\title{
Clinical Outcomes of First-line Abiraterone Acetate or Enzalutamide for Metastatic Castration-resistant Prostate Cancer After Androgen Deprivation Therapy + Docetaxel or ADT Alone for Metastatic Hormone-sensitive Prostate Cancer
}

\author{
Edoardo Francini, ${ }^{1,2}$ Steven Yip, ${ }^{3}$ Shubidito Ahmed, ${ }^{3}$ Haocheng Li, ${ }^{4}$ \\ Luke Ardolino, ${ }^{5}$ Carolyn P. Evan, ${ }^{1}$ Marina Kaymakcalan, ${ }^{1}$ Grace K. Shaw, ${ }^{1}$ \\ Philip W. Kantoff, ${ }^{6}$ Mary-Ellen Taplin, ${ }^{1}$ Nimira S. Alimohamed, ${ }^{3}$ \\ Anthony M. Joshua, ${ }^{5}$ Daniel Y.C. Heng, ${ }^{3}$ Christopher J. Sweeney ${ }^{1}$
}

\begin{abstract}
Although an androgen deprivation therapy plus docetaxel regimen was shown to extend the survival of some patients with metastatic hormone-sensitive prostate cancer compared with androgen deprivation therapy alone, currently, there is no report in the literature investigating the impact of upfront docetaxel on subsequent first-line abiraterone acetate or enzalutamide for metastatic castration-resistant prostate cancer. This study showed that their activity is maintained regardless of previous use of docetaxel for metastatic hormonesensitive prostate cancer. These findings can aid treatment decision-making.

Background: The CHAARTED (ChemoHormonal Therapy Versus Androgen Ablation Randomized Trial for Extensive Disease in Prostate Cancer) and STAMPEDE (Systemic Therapy in Advancing or Metastatic Prostate Cancer: Evaluation of Drug Efficacy) trials showed that the addition of docetaxel (D) to androgen deprivation therapy (ADT) prolonged longevity of men with metastatic hormone-sensitive prostate cancer (mHSPC). However, the impact of upfront $D$ on subsequent therapies is still unexplored. As abiraterone acetate (AA) and enzalutamide (E) are the most commonly used first-line treatment for metastatic castration-resistant prostate cancer (mCRPC), we aimed to assess whether they maintained their efficacy after ADT+D versus ADT alone. Patients and Methods: A cohort of patients with $\mathrm{MCRPC}$ treated between 2014 and 2017 with first-line AA or E for mCRPC was identified from 3 hospitals' institutional review board-approved databases. Patients were classified by use of $D$ for $\mathrm{mHSPC}$. This time frame was chosen as ADT+D became a valid therapeutic option for mHSPC in 2014, and it inherently entailed a short follow-up time on AA/E. The endpoints included overall survival from ADT start, overall survival from AA/E start, and time to AAVE start from ADT start. Differences between groups were assessed using the log-rank test. Results: Of the 102 patients with mCRPC identified, $50(49 \%)$ had previously received ADT alone, while $52(51 \%)$ had ADT+D. No statistically significant difference in any of the evaluated outcomes was observed between the 2 cohorts. Yet, deaths in the ADT+D group were 12 versus 21 in the ADT alone, after a median follow-up of 24.4 and 29.8 months, respectively. Conclusion: In a cohort of ADT/ADT+D-treated patients with mCRPC with short times to first-line AA/E and follow-up, the efficacy of AA/E is similar regardless of previous use of $D$.
\end{abstract}

Clinical Genitourinary Cancer, Vol. 16, No. 2, 130-4 (c) 2017 Elsevier Inc. All rights reserved.

Keywords: AR targeting agents; CHAARTED, mCRPC; mCRPC sequencing; mHSPC

${ }^{1}$ Dana-Farber Cancer Institute, Harvard Medical School, Boston, MA

${ }^{2}$ Sapienza Università di Roma, Rome, Italy

${ }^{3}$ Tom Baker Cancer Centre, Calgary, AB, Canada

${ }^{4}$ Departments of Oncology and Community Health Sciences, University of Calgary,

Calgary, AB, Canada

${ }^{5}$ St Vincent's Hospital, Sydney, Australia

${ }^{6}$ Memorial Sloan Kettering Cancer Center, New York, NY
Submitted: Oct 31, 2017; Accepted: Dec 17, 2017; Epub: Dec 27, 2017

Address for correspondence: Christopher J. Sweeney, MBBS, Lank Center for Genitourinary Oncology, Dana-Farber Cancer Institute, 450 Brookline Ave, Boston, MA 02215

E-mail contact: christopher_sweeney@dfci.harvard.edu 


\section{Introduction}

Until recently, androgen deprivation therapy (ADT) by surgical or medical castration has been the standard-of-care for metastatic hormone-sensitive prostate cancer (mHSPC). ${ }^{1}$ Upon progression, prostate cancer is termed castration-resistant, and chemotherapy and second-generation androgen receptor (AR) targeting agents, such as abiraterone acetate (AA) and enzalutamide $(\mathrm{E})$, proven in clinical trials to increase longevity of men with metastatic castrationresistant prostate cancer ( $\mathrm{mCRPC}$ ), are used in patients deemed fit. $^{2-4}$ In this regard, a recent analysis of prescriptions in the United States reported that AA and E are the preferred (76\%) first-line therapy for men with mCRPC.

Recently, 2 new regimens combining ADT with docetaxel (D) and AA, respectively, have shown a survival advantage compared with $\mathrm{ADT}$ alone and have become new valid therapeutic options for the patient with mHSPC. ${ }^{6-9}$ Particularly, the chemohormonal combination showed a clear benefit for men with mHSPC with high-volume disease compared with ADT alone and has been introduced in the treatment paradigm of $\mathrm{mHSPC}$ in 2014. However, to date, there is no report in the literature evaluating the impact of early $\mathrm{D}$ on subsequent first-line AA or E for mCRPC, and an optimal sequence of drugs after progression on $\mathrm{ADT}+\mathrm{D}$ does not exist. The E3805 CHAARTED (ChemoHormonal Therapy Versus Androgen Ablation Randomized Trial for Extensive Disease in Prostate Cancer) investigators demonstrated that the addition of $\mathrm{D}$ to $\mathrm{ADT}$ versus $\mathrm{ADT}$ alone prolonged time to CRPC (19.4 vs. 11.7 months; hazard ratio [HR], 0.61; 95\% confidence interval $[\mathrm{CI}], 0.52-0.73 ; P<.0001)$, and time to clinical progression (33.0 vs. 19.8 months; HR, 0.62; 95\% CI, 0.51-0.75; $P<.0001)$, respectively. ${ }^{6}$ Based on the similar HR data, we postulated that first-line AA or E for mCRPC at least maintained efficacy after $\mathrm{ADT}+\mathrm{D}$ versus ADT alone. The biological rationale for this hypothesis is that early D debulks AR-independent tumor cells that would otherwise thrive under the positive selective pressure exerted by $\mathrm{ADT}$ alone and thus enhances the efficacy of AR-targeting AA or $\mathrm{E}$ as first-line treatment for mCRPC. ${ }^{6,10}$ Determining the impact of early $\mathrm{D}$ for $\mathrm{mHSPC}$ on subsequent AA/E therapy would greatly aid treatment decision-making and provide useful data to delineate the most appropriate sequence of drugs for a given patient after failure of the chemohormonal regimen. Therefore, in this report, we aimed to evaluate the outcomes of first-line AA or E treatment for patients with mCRPC selected from 3 hospitals' databases and classified by use of D for $\mathrm{mHSPC}$.

\section{Patients and Methods}

From the Dana-Farber Cancer Institute of Boston, the St Vincent's Hospital of Sydney, and the Tom Baker Cancer Centre of Calgary Institutional Review Board-approved databases, we retrospectively identified consecutive patients with histologically confirmed and radiologically evaluable mCRPC who had initiated $\mathrm{AA}$ or $\mathrm{E}$ as first-line treatment for mCRPC between 2014 and 2017. This time frame was selected considering that the chemohormonal regimen was recognized as a valid therapeutic option for mHSPC in 2014. Patients were excluded if they had received AA or E for any earlier stage of disease or had been given any other agent as first-line treatment for mCRPC. The final cohort was classified by previous use of $\mathrm{D}$ for $\mathrm{mHSPC}$ into 2 groups: $\mathrm{ADT}+\mathrm{D}$ and $\mathrm{ADT}$ alone. The primary endpoints of the study were overall survival (OS) from ADT start and OS from $\mathrm{AA} / \mathrm{E}$ start, defined as time from ADT for $\mathrm{mHSPC}$ and time from first-line AA/E for mCRPC start, respectively, to death from any cause or censored at last follow-up visit. Time to $\mathrm{AA} / \mathrm{E}$ start, defined as the interval from $\mathrm{ADT}$ commencement to initiation of first-line AA/E for mCRPC, was the secondary endpoint. The Kaplan-Meier method was used to estimate the distributions of the endpoints, including median time-toevent and its $95 \% \mathrm{CI}$, and the log-rank test was used to compare time-to-event distributions between the 2 groups. Time of metastatic disease presentation, whether after prior definitive local therapy or newly diagnosed (de novo), biopsy Gleason score, and follow-up data was collected from medical records.

\section{Results}

Overall, 102 patients with $\mathrm{mCRPC}$ were eligible for this analysis. Fifty-two (51\%) had received ADT+D and 50 (49\%) ADT alone, for mHSPC. Baseline patient characteristics are described in

Table 1 Patient Characteristics According to the Use of D in Addition to ADT for mHSPC

\begin{tabular}{|c|c|c|c|}
\hline Characteristics & Total, $N=102(\%)$ & ADT Alone, $\mathbf{N}=50(\%)$ & $A D T+D, N=52(\%)$ \\
\hline \multicolumn{4}{|l|}{ Biopsy Gleason score } \\
\hline$\leq 6$ & $1(1)$ & $1(2)$ & $0(0)$ \\
\hline 7 & $15(15)$ & $1(2)$ & $14(27)$ \\
\hline$\geq 8$ & $37(36)$ & $23(46)$ & $14(27)$ \\
\hline Missing data & $49(48)$ & $25(50)$ & $24(46)$ \\
\hline Prior local therapy & $30(29)$ & $15(30)$ & $15(29)$ \\
\hline De novo & $72(71)$ & $35(70)$ & $37(71)$ \\
\hline \multicolumn{4}{|l|}{ First-line AA/E } \\
\hline AA & $51(50)$ & $24(48)$ & $27(52)$ \\
\hline $\mathrm{E}$ & $48(47)$ & $23(46)$ & $25(48)$ \\
\hline $\mathrm{AA}+\mathrm{E}$ & $3(3)$ & $3(6)$ & $0(0)$ \\
\hline Median follow-up, mos (95\% Cl) & $26.4(24.0-29.9)$ & $29.8(25.1-31.4)$ & $24.4(21.7-27.1)$ \\
\hline
\end{tabular}

Abbreviations: $\mathrm{AA}=$ Abiraterone acetate; $\mathrm{ADT}=$ androgen deprivation therapy; $\mathrm{Cl}=$ confidence interval; $\mathrm{D}=$ docetaxel; $\mathrm{E}=$ enzalutamide; $\mathrm{mHSPC}=$ metastatic hormone sensitive prostate cancer; mos $=$ months; $\mathrm{N}=$ number. 
Table 2 Outcomes According to the Use of D in Addition to ADT for mHSPC

\begin{tabular}{|c|c|c|c|c|c|c|c|c|}
\hline $\begin{array}{l}\text { First-line } \\
\text { AA/E }\end{array}$ & N (\%) & $\begin{array}{l}\text { No. Deaths (Median } \\
\text { Follow-up, mos) }\end{array}$ & $\begin{array}{l}\text { Median OS From } \\
\text { ADT Start, } \\
\text { mos (95\% Cl) }\end{array}$ & $P$ Value & $\begin{array}{l}\text { Time to AA/E Start, } \\
\text { mos }(95 \% \mathrm{Cl})\end{array}$ & $P$ Value & $\begin{array}{c}\text { Median OS } \\
\text { From AA/E Start, } \\
\text { mos }(95 \% \text { Cl) }\end{array}$ & $P$ Value \\
\hline ADT & $50(49)$ & $21(29.8)$ & 33.5 (22.4 to NR) & .2047 & $11.0(8.5-13.7)$ & .7265 & 17.3 (13.7 to NR) & .6514 \\
\hline$A D T+D$ & $52(51)$ & $12(24.4)$ & NR (NR to NR) & & $12.8(11.1-15.7)$ & & NR (13.1 to NR) & \\
\hline
\end{tabular}

Abbreviations: $\mathrm{AA}=$ Abiraterone acetate; $\mathrm{ADT}=$ androgen deprivation therapy; $\mathrm{Cl}=$ confidence interval; $\mathrm{D}=$ docetaxel; $\mathrm{E}=$ enzalutamide; mos $=$ months; No = number; $\mathrm{NR}=$ not yet reached; OS = overall survival.

Table 1. Despite randomization not being part of the study design, the 2 cohorts were well-balanced in terms of prior local therapy/de novo, median follow-up, and distributions of AA and E, which were similar also in the total population (50\% vs. $47 \%$, respectively). Most patients $(71 \%)$ had de novo disease, and of the 53 with available data, 37 had biopsy Gleason score $\geq 8$. Median follow-up was only 26.4 months (95\% CI, 24.0-29.9 months) owing to the chosen eligibility time window. For the same reason, time to $\mathrm{AA} / \mathrm{E}$ start was also short at 11.0 months (95\% CI, 8.5-13.7 months) for the ADT-alone cohort and 12.8 months (95\% CI, 11.1-15.7 months) for the $\mathrm{ADT}+\mathrm{D}$ cohort (Table 2). In this regard, no statistically significant difference was observed in time to AA/E start between the 2 study groups $(P=.7265)$. Furthermore, the 2 cohorts did not show any significant difference in OS from ADT start $(P=.2047)$ or in OS from AA/E start $(P=.6514)$ (Table 2). In the $\mathrm{ADT}+\mathrm{D}$ group, neither the median survival from ADT start (not yet reached [NR]; 95\% CI, NR to NR) nor the median OS from AA/E start (NR; 95\% CI, 13.1 to NR) had been reached at the time of the analysis. The Kaplan-Meier estimates confirm these findings, showing the 2 study groups with similar survival and time to AA/E start curves, respectively, and not-reached median survivals for the chemohormonal cohort (Figure 1). Notably, OS from ADT start in the ADT alone group at 33.5 months $(95 \% \mathrm{CI}, 22.4$ months to NR) was in line with that of patients with poor prognostic factors, such as high-volume and de novo metastatic disease, as noted in the CHAARTED, LATITUDE (Study of Abiraterone Acetate Plus Low-Dose Prednisone Plus Androgen Deprivation Therapy (ADT) versus ADT Alone in Newly Diagnosed Participants With HighRisk, Metastatic Hormone-Naive Prostate Cancer), and GETUG (Genitourinary Group)-AFU (French Association of Urology) 15 trials. ${ }^{8,11,12}$ In this respect, prostate-cancer deaths in the ADT-alone group were 21 versus 12 in the $\mathrm{ADT}+\mathrm{D}$ group, after a median follow-up of 29.8 and 24.4 months, respectively (Table 2).

\section{Discussion}

Although the addition of $\mathrm{D}$ to ADT proved to be significantly beneficial for men with mHSPC, ${ }^{6,7}$ the impact of upfront $\mathrm{D}$ on later therapies has not been yet investigated, and an optimal treatment sequence for mCRPC has not been defined by well-powered prospective trials, even for patients receiving $\mathrm{D}$ in the standard setting. In a recent analysis, second-generation hormonal agents AA and $\mathrm{E}$ have been reported as the most commonly administered firstline treatment for men in the United States with mCRPC. ${ }^{5}$ To our knowledge, this retrospective study is the first to explore the influence of early $\mathrm{D}$ on the clinical efficacy of subsequent first-line AA or $\mathrm{E}$ for mCRPC, using contemporaneous cohorts of $\mathrm{ADT}+\mathrm{D}$ and ADT-alone pretreated men with mCRPC. It has been theorized that early chemotherapy disposes of AR-independent tumor clones;

\section{Figure 1 Kaplan-Meier Estimates of OS From ADT Start (A), OS From AA/E Start (B), and Time to AA/E Start (C)}

A

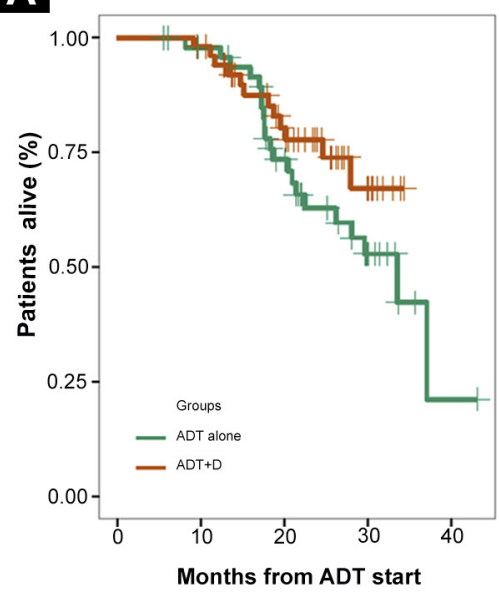

B

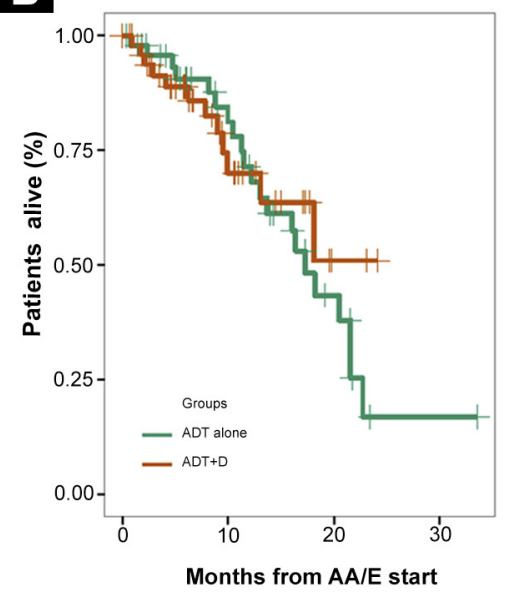

C

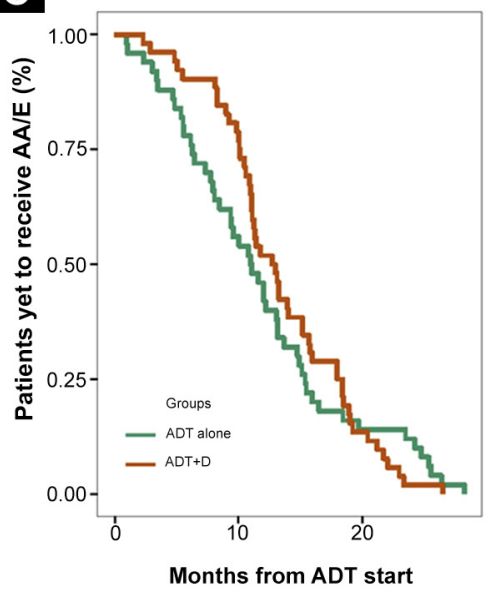

Abbreviations: $\mathrm{AA}=$ Abiraterone acetate; $\mathrm{ADT}=$ androgen deprivation therapy; $\mathrm{E}=$ enzalutamide; $\mathrm{OS}=$ overall survival. 
hence, favoring efficacy of AR targeting AA/E as first-line treatment for mCRPC. ${ }^{6,10}$ On the other hand, a few in vivo and in vitro studies observed cross-resistance between $\mathrm{D}$ for $\mathrm{mCRPC}$, and subsequent $\mathrm{AA}$ or $\mathrm{E}$ and partly attributed it to the partially overlapping mechanisms of action of these drugs, which interfere with AR signaling pathway and inhibit AR nuclear translocation. ${ }^{13-15}$ In the present study, D is used for the hormone-sensitive disease, but the treatment sequence is the same; hence, cross-resistance could arise. However, this report showed that first-line AA or E therapy for mCRPC maintains its activity whether $\mathrm{D}$ was used upfront or not. Specifically, there was no statically significant difference in any of the explored outcomes. Nonetheless, it should be noted that, in the ADT-alone group, OS from AA/E start was considerably shorter at 17.3 months ( $95 \% \mathrm{CI}, 13.7$ months to NR) than observed with pre-chemotherapy $\mathrm{AA}$ and $\mathrm{E}$ for $\mathrm{mCRPC}$ in the final analyses of their pivotal trials (34.7 months [95\% CI, 32.7-36.8 months] and 35.3 months [95\% CI, 32.2 months to NR], respectively). ${ }^{16,17}$ Furthermore, OS from ADT start was short at 33.5 months (95\% CI, 22.4 months to NR) and consistent with a population with poor prognostic features. ${ }^{8,11,12}$ Patients' baseline characteristics seem to support this premise, because $71 \%$ of the total population and $70 \%$ of the ADT alone cohort had de novo metastatic disease, and 37 of the 53 patients without missing data and, remarkably, 23 of 25 in the ADT-alone cohort had biopsy Gleason score $\geq 8$. This is not surprising given the chosen recruitment time window, which inevitably determined a short median follow-up and a selection of patients with short times to AA/E start, which were 29.8 months (95\% CI, 25.1-31.4 months) and 11.0 months (95\% CI, 8.5-13.7 months) for the ADT-alone cohort, respectively. The present analysis is therefore heavily weighted by the early events, which were very similar in both arms, even in the pivotal studies that, as a matter of fact, required a longer follow-up for the benefits to be seen. As such, although the median survivals in the $\mathrm{ADT}+\mathrm{D}$ group were not reached at the time of the analysis, there was no statistically significant difference from the ADT-alone group. Yet prostate cancer deaths were more numerous in the ADT-alone cohort compared with the $\mathrm{ADT}+\mathrm{D}$ cohort (21 vs. 12 after a median follow-up of 29.8 and 24.4 months, respectively). This finding is visually highlighted by the Kaplan-Meier estimates of OS from ADT start, which show a gap between the curves starting after 15 months and quite steadily widening over time in favor of the combination group (Figure 1A). This also appears to be the case of OS from AA/E start (Figure 1B). Longer follow-up and possibly larger samples would be needed to confirm this trend. However, despite not reaching a statistically significant difference, men treated with the chemohormonal regimen tended to experience a longer time to AA/E start (Figure 1C) compared with those treated with ADT alone. Therefore, considering that the 2 study groups were well-balanced with respect to the number of patients and the clinical and treatment characteristics, we could speculate that patients who received upfront $\mathrm{D}$ appear to be faring better with first-line $\mathrm{AA}$ or $\mathrm{E}$ for mCRPC than patients who received ADT alone, which is consistent with our hypothesis. Larger prospective studies with longer follow-up and better biological and clinical patient characterization would be needed to confidently confirm this hypothesis.

As the chemohormonal regimen is being increasingly administered to the population with mHSPC, gathering evidence on the most appropriate sequence of additional treatments after failure of upfront $\mathrm{D}$ has become paramount. To the authors' knowledge, the present analysis is the first to provide useful clinical data to address this issue. Nevertheless, the innate biases of this study, including the short median follow-up, small sample size, and retrospective nature, represent limitations that prevent generalization of the results. On the other hand, interpreted with caution, this data may aid the physician in treatment decision-making and reassure him on the use of early D for mHSPC. In fact, our outcomes showed that exposure to upfront $\mathrm{D}$ for mHSPC patients with poor prognostic factors, such as de novo metastatic presentation, at least does not reduce the efficacy of later AA/E used as first-line treatment for mCRPC. This conclusion is supported by the results of the latest post-hoc analysis of the STAMPEDE (Systemic Therapy in Advancing or Metastatic Prostate Cancer: Evaluation of Drug Efficacy) trial comparing 2 contemporaneously randomized cohorts of patients treated with $\mathrm{ADT}+\mathrm{D}$ and $\mathrm{ADT}+\mathrm{AA}$, respectively. ${ }^{18}$ Despite the limited statistical power and, similar to our study, a short follow-up (approximately 4 years), this report showed that the ADT plus AA significantly extends failure-free survival and progression-free survival compared with the chemohormonal regimen in the population with mHSPC. However, OS from randomization did not differ between the 2 treatment arms. Because most patients progressing after upfront $\mathrm{D}$ received $\mathrm{AA}$ or $\mathrm{E},{ }^{18}$ it would appear that the activity of first-line AA or E for mCRPC was at least not diminished by previous use of $\mathrm{D}$, as suggested by our analysis.

Given the heterogeneity of prostate cancer, we deem it unlikely that a single sequence of treatments could provide the best benefit/ risk ratio to all patients with $\mathrm{mCRPC}$ progressing on early D. An individualized therapeutic approach that takes into account the diverse tumor biological and molecular features, patient clinical characteristics, and prognostic and predictive biomarkers would be required to maximize survival. However, although the continuous advances in the knowledge of tumor biology and in the development of biotechnologies pave the way towards a future of precision medicine, clinical reports addressing treatment sequencing questions can provide the clinician with useful indications to make evidencebased decisions.

\section{Conclusion}

Despite the shortcomings of this analysis, we deem our data clinically relevant as they suggest that, in a cohort of patients with short median follow-up and time to $\mathrm{AA} / \mathrm{E}$ start, first-line $\mathrm{AA} / \mathrm{E}$ treatment for mCRPC maintains its efficacy regardless of previous use of $\mathrm{D}$ for $\mathrm{mHSPC}$.

\section{Clinical Practice Points}

- AA and E are the most frequently used first-line treatment for mCRPC.

- In patients with mCRPC, the efficacy of $\mathrm{AA} / \mathrm{E}$ seems to be similar regardless of previous use of D for mHSPC.

- Larger prospective studies with longer follow-up and better biological and clinical patient characterization are warranted to confirm these findings.

- These results may aid the physician in the treatment decisionmaking and reassure him on the use of early $\mathrm{D}$ for mHSPC. 


\section{Disclosure}

PWK received compensation for scientific advisory board/ consulting for Astellas, Bayer, Bellicum, BIND Biosciences, BN Immunotherapeutics, DRGT, Ipsen Pharmaceuticals, Janssen, Metamark, Merck, MTG Therapeutics, New England Research Institutes, Omnitura, OncoCellMDX, Progenity, Sanofi, Tarveda Therapeutics, Thermo Fisher Scientific; he has investment interests in Bellicum, DRGT, Metamark, Tarveda Therapeutics; and he is on data safety monitoring board of Genetech/Roche, Merck, Oncogenex. $\mathrm{LCH}$ reports compensation for consulting/advisory role at Dendreon, Genentech, KEW Group, Medivation/Astellas, Pfizer, Theragene; and institutional research funding from Bayer, Dendreon, Genentech/Roche, Medivation/Astellas, Sotio, Takeda, BMS; Travel, Accommodations, and Expenses from Sanofi. M-ET reports personal fees for attending advisory boards for Janssen and Medivation; and receives research funding from Janssen and Medivation. DYCH declares compensation for advisory board for Astellas and Janssen. CJS reports consulting with compensation for Astellas, Bayer, Genentech, Janssen, Pfizer, Sanofi; and received research funding from Astellas, Janssen, Sotio, Sanofi. The remaining authors have stated that they have no conflicts of interest.

\section{References}

1. Huggins C, Stevens RE Jr, Hodges CV. Studies on prostatic cancer: II. The effects of castration on advanced carcinoma of the prostate gland. Arch Surg 1941; 43: 209-23.

2. Tannock IF, de Wit R, Berry WR, et al, TAX 327 Investigators. Docetaxel plus prednisone or mitoxantrone plus prednisone for advanced prostate cancer. $N$ EnglJ Med 2004; 351:1502-12.

3. Petrylak DP, Tangen CM, Hussain MH, et al. Docetaxel and estramustine compared with mitoxantrone and prednisone for advanced refractory prostate cancer. N Engl J Med 2004; 351:1513-20.

4. Shiota M, Yokomizo A, Fujimoto N, Kuruma H, Naito S. Castration-resistant prostate cancer: novel therapeutics pre- or post-taxane administration. Curr Cancer Drug Targets 2013; 13:444-59.
5. Flaig TW, Potluri RC, Ng Y, Todd MB, Mehra M. Treatment evolution for metastatic castration-resistant prostate cancer with recent introduction of novel agents: retrospective analysis of real-world. Cancer Med 2016; 5:182-91.

6. Sweeney CJ, Chen YH, Carducci M, et al. Chemohormonal therapy in metastatic hormone-sensitive prostate cancer. N Engl J Med 2015; 373:737-46.

7. James ND, Sydes MR, Clarke NW, et al, STAMPEDE investigators. Addition of docetaxel, zoledronic acid, or both to first-line long-term hormone therapy in prostate cancer (STAMPEDE): survival results from an adaptive, multiarm, multistage, platform randomised controlled trial. Lancet 2016; 387:1163-77.

8. Fizazi K, Tran NP, Fein L, et al, LATITUDE Investigators. Abiraterone plus prednisone in metastatic, castration-sensitive prostate cancer. N Engl J Med 2017 377:352-60.

9. James ND, de Bono JS, Spears MR, et al, STAMPEDE Investigators. Abiraterone for prostate cancer not previously treated with hormone therapy. $N$ Engl J Med 2017; 377:338-51.

10. Ahmed M, Li LC. Adaptation and clonal selection models of castration-resistant prostate cancer: current perspective. Int J Urol 2013; 20:362-71.

11. Sweeney CJ, Chen YH, Liu G, et al. Long term efficacy and QOL data of chemohormonal therapy (C-HT) in low and high volume hormone naïve metastatic prostate cancer (PrCa): E3805 CHAARTED trial. Ann Oncol 2016; 27:243-65.

12. Gravis G, Boher JM, Joly F, et al, GETUG. Androgen Deprivation Therapy (ADT) Plus Docetaxel Versus ADT Alone in Metastatic Non castrate Prostate Cancer: Impact of Metastatic Burden and Long-term Survival Analysis of the Randomized Phase 3 GETUG-AFU15 Trial. Eur Urol 2016; 70:256-62.

13. Nadal R, Zhang Z, Rahman H, et al. Clinical activity of enzalutamide in docetaxelnaïve and docetaxel-pretreated patients with metastatic castration-resistant prostate cancer. Prostate 2014; 74:1560-8.

14. van Soest RJ, van Royen ME, de Morrée ES, et al. Cross-resistance between taxanes and new hormonal agents abiraterone and enzalutamide may affect drug sequence choices in metastatic castration-resistant prostate cancer. Eur I Cancer 2013; 49:3821-30.

15. Hoshi S, Numahata K, Ono K, et al. Treatment sequence in castration-resistant prostate cancer: a retrospective study in the new anti-androgen era. Mol Clin Oncol 2017; 7:601-3.

16. Ryan CJ, Smith MR, Fizazi K, et al, COU-AA-302 Investigators. Abiraterone acetate plus prednisone versus placebo plus prednisone in chemotherapy-naive men with metastatic castration-resistant prostate cancer (COU-AA-302): final overall survival analysis of a randomised, double-blind, placebo-controlled phase 3 study. Lancet Oncol 2015; 16:152-60.

17. Beer TM, Armstrong AJ, Rathkopf D, et al. Enzalutamide in men with chemotherapy-naïve metastatic castration-resistant prostate cancer: extended analysis of the phase 3 PREVAIL study. Eur Urol 2017; 71:151-4.

18. Sydes MR, Mason MD, Spears MR, et al. Adding abiraterone acetate plus prednisolone (AAP) or docetaxel for patients (pts) with high-risk prostate cancer (PCa) starting long-term androgen deprivation therapy (ADT): directly randomised data from STAMPEDE (NCT00268476). Ann Oncol 2017; 28:605-49. 